\title{
Chronic unpredictable stress inhibits nociception in male rats
}

\author{
Filipa Pinto-Ribeiro, Armando Almeida*, José M. Pêgo, João Cerqueira, Nuno Sousa \\ Life and Health Sciences Research Institute, School of Health Sciences, University of Minho, Braga, Portugal \\ Received 21 November 2003; received in revised form 16 January 2004; accepted 8 February 2004
}

\begin{abstract}
Chronic stress elicits remarkable alterations to the structure and function of several areas of the central nervous system. Nociception is known to be affected by chronic stress and age, although the observations are contradictory. Herein we report that both young and old rats submitted to a chronic unpredictable stress paradigm have reduced nociception in the tail-flick nociceptive test. Moreover, stressed animals show an increase in nociceptive threshold after three successive exposures to noxious stimulation (within a 2 min interval). While the sustained stress-induced analgesia is usually attributed to the resulting hypercorticalism, the immediate exacerbation of tolerance to pain displayed by stressed animals is most likely mediated through other mechanisms due to the very rapid antinociceptive effect observed. (C) 2004 Elsevier Ireland Ltd. All rights reserved.
\end{abstract}

Keywords: Rat; Chronic unpredictable stress; Pain tolerance; Tail-flick; Antinociception

Tissue injury and inflammation are associated with persistent stimulation of nociceptors, which transmit the nociceptive input from the periphery to second-order neurons located in the spinal cord dorsal horn [12]. The transmission of nociceptive messages at the spinal level can be modulated by the supraspinal pain control system through endogenous mechanisms mediated by descending projections to the spinal dorsal horn $[8,12]$. Recent studies have shown that these mechanisms involve the descending facilitation or inhibition of spinal nociceptive transmission triggered by the activation of supraspinal pronociceptive or antinociceptive areas [9,12], respectively. Thus, it is probable that pain may result from the balance between opposing supraspinal modulatory actions impinging upon dorsal horn neurons mediating the transmission of nociceptive information to the brain [12].

Nociception can also be modulated by external factors through alterations in intrinsic pain pathways. Exposure to acute stress is amongst these factors, as it is known to produce analgesia [4]. Yet, nociceptive modulation following prolonged exposure to stress is still a matter of dispute, with some studies reporting an increased pain threshold [1] while others demonstrate hyperalgesia [4]. While the

\footnotetext{
* Corresponding author. Escola de Ciências da Saúde, Universidade do Minho, Complexo Pedagógico II, Piso 3, Campus de Gualtar, 4710-057 Braga, Portugal. Tel.: + 351-253-604-808; fax: + 351-253-604-809.

E-mail address: aalmeida@ecsaude.uminho.pt (A. Almeida).
}

mechanisms involved in these phenomena remain cryptic, it is well known that prolonged exposure to stressful stimuli activates the hypothalamo-pituitary-adrenocortical axis (HPA), resulting in sustained elevations of corticosterone (the dominant endogenous glucocorticoid in rodents) [6].

During their lives organisms are challenged with various stressors and the ability to handle them varies considerably with age [15]. There is substantial evidence that aging impacts on neuroendocrine stress responses, and also in their deleterious consequences upon brain structure and function [15]. In addition, age differences in the sensitivity to phasic nociceptive stimulation have also been reported but show contradictory results: there are studies demonstrating that acute nociceptive thresholds increase [13], decrease [2] and do not change [5] with age. Moreover, inconsistent data are also reported concerning the response to tonic painful stimulation and pain inhibitory actions [3].

The present study aims to characterize the influence of chronic stress on nociception with a triple purpose: to evaluate the effect on (i) pain perception (nociceptive threshold) and (ii) tolerance to pain (variation of the nociceptive threshold following a short successive exposition to the same noxious stimulation), and to analyze (iii) the influence of age on both pain perception and tolerance to pain. A model of chronic unpredictable stress was chosen in order to avoid habituation to the stressor and to maintain robust the stress response throughout the experiment [16]. 
All regulations determined by the local veterinarian committee (in accordance with the European Community Council Directive 86/609/EEC) concerning the handling of laboratory animals and the international ethical guidelines for the study of experimental pain in conscious animals [19] were followed. Adult male Wistar rats (Charles Rivers, Barcelona, Spain) were assigned to one of the following four groups: (i) young non-stressed controls (YNS) $(n=8$; 3 months old) and (ii) old non-stressed controls (ONS) ( $n=8 ; 18$ months old) - both groups were maintained under standard laboratory conditions; (iii) young chronic stressed animals (YCS) $(n=8 ; 3$ months old) and (iv) old chronic stressed animals (OCS) $(n=8 ; 18$ months old) both groups were submitted during a period of 21 days to a chronic unpredictable stress paradigm that consisted of applying one of several stressors (one stressor per day) in a random order: forced swimming (three times in a $3 \mathrm{~min}$ bath with a $2 \mathrm{~min}$ rest between each; water at $\pm 20{ }^{\circ} \mathrm{C}$ ), overcrowding $(1 \mathrm{~h})$, restraint $(30 \mathrm{~min})$ and placement on a vibrating/rocking platform (1 h). As shown previously [16], this stress inducing regimen results in chronically elevated serum corticosterone titers throughout the experimental period.

Alteration in pain perception was studied using the tailflick (TF) test (Model 7360, Ugo Basile, Comerio, Italy), which is used to assess reflexive nociceptive thresholds. Rats were tested on day 21 at 11:00 a.m. by being submitted to three TF tests within a 2 min interval in order to test for tolerance to pain stimulation. To avoid bias related to the handling and testing of the rats, a 1 week period prior to the first nociceptive test was established for the habituation of the animals to the behavioral test equipment. Animals were placed daily in the test room for $2 \mathrm{~h}$ followed by a $1 \mathrm{~min}$ handling and $1 \mathrm{~min}$ training session in the TF apparatus (without performing the test).

The statistical evaluation of the results was made as follows: (a) the nociceptive behavior in old/young rats in stressed/non-stressed groups was analyzed by performing a two-way ANOVA (considering age and treatment as independent variables); (b) the variations of TF latencies trial by trial (from the first to the third trial) were analyzed by Student's $t$-test on young and old groups of animals. Differences between means were considered statistically significant when $P<0.05$.

Chronic stress induced significant increases in $\mathrm{TF}$ latencies at two levels: (a) between the mean TF latency of non-stressed and stressed animals; and (b) between the first and third TF tests of stressed animals. In fact, TF latency increased significantly between YNS and YCS animals (Fig. 1) and between ONS and OCS animals [twoway ANOVA, $F_{(1)}=14,567, P<0.001$ ] (Fig. 1). Importantly, the analysis of TF latencies trial by trial also revealed a significant increase in the nociceptive threshold from the first to the third trial in both young and old stressed animals ( $t$-tests, $P=0.016$ and $P=0.027$, respectively) but not in the control groups ( $t$-tests, $P=0.99$ and $P=0.93$,

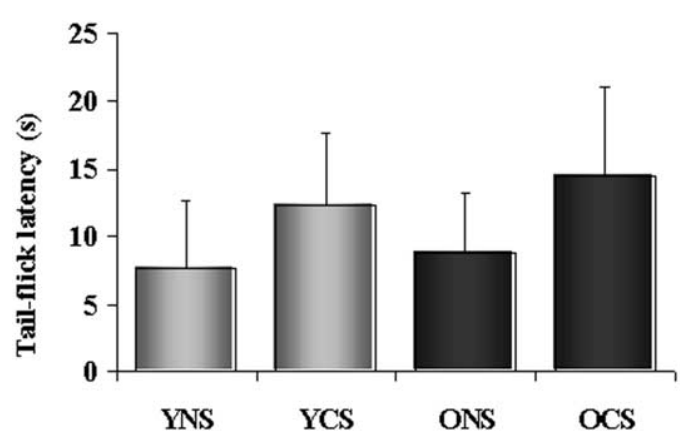

Fig. 1. Effect of chronic unpredictable stress on the nociceptive behavior of YNS and YCS rats and of ONS and OCS animals. Mean TF latency on day 21 of treatment. Mean \pm SD.

respectively) (Fig. 2). The ANOVA analysis failed to reveal any effect of age on nociceptive threshold $\left[F_{(1)}=1,355\right.$, $P=0.24]$.

Overall, this study shows that chronic unpredictable stress inhibits pain-like behavior by increasing the nociceptive threshold. Moreover, the strong analgesia observed from the first to the third noxious stimulation presented in a very short period of time, in animals never tested before, illustrates a remarkable tolerance to a painful stimulus induced by this model of chronic stress. Finally, this helplessness-like effect of chronic stress on pain tolerance was demonstrated in both young and old animals.

Individuals exposed to stressful conditions show an increased nociceptive threshold, known as stress-induced analgesia, which may involve a different neurochemical basis related to stress severity [18]. Consistent with these findings, we have observed that stressed rats (either young
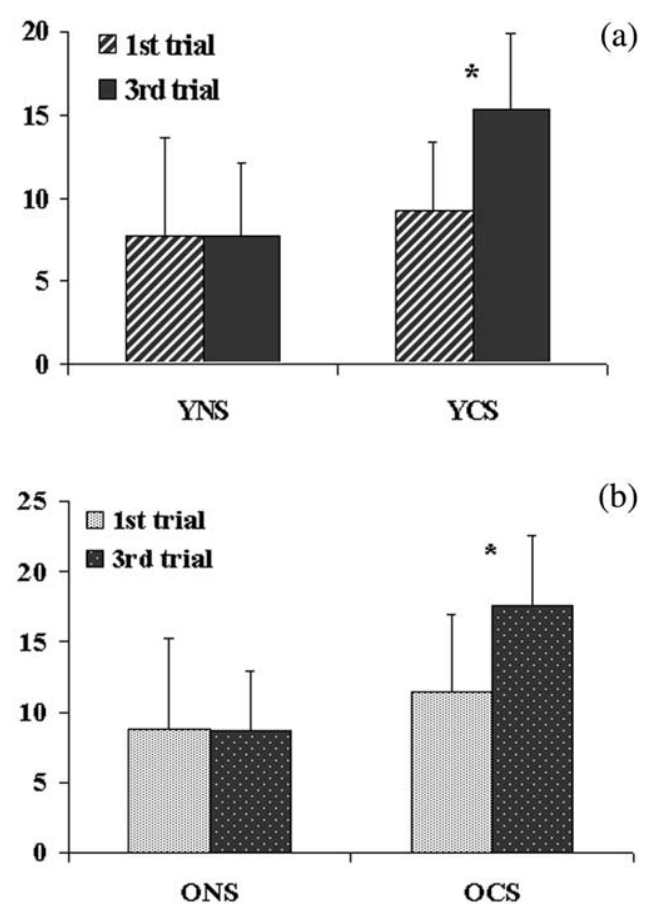

Fig. 2. Mean TF latency on day 21 of the first and third successive TF tests applied to young (a) and old (b) animals. Mean \pm SD. $* P<0.05$. 
or old) show higher TF latencies when compared to unstressed controls. Although the influence of stress upon pain perception has been extensively reproduced in several laboratories using different acute stress paradigms [18], the same tests in chronic stressed animals produced contradictory results: some studies report decreased pain perception in chronically stressed subjects [1], while others demonstrate the opposite effect (hyperalgesia) [4]. This apparent paradox might, at least partially, result from differences in the experimental procedures: indeed, whilst studies reporting stress-induced analgesia presented repeatedly the same stressor, others (like the one herein used) presented multiple stressors in an unpredictable paradigm. One likely consequence of these two different protocols (single vs. multiple stressors) is a remarkable difference in the development of habituation to stress $[4,14]$; indeed, repeated exposure to the same aversive stimulus leads to an attenuated stress response in these subjects [10], while this response is still robust in those facing unpredictable paradigms [16].

Besides the maintenance of robust stress responses during the entire experimental process, chronically stressed animals displayed increased tolerance to pain, that is, a decreased nociceptive reaction to painful stimulation. Indeed, the re-exposure twice to a TF test in the $2 \mathrm{~min}$ after the first exposure produced a remarkable increase in latency in chronically stressed animals; although agerelated differences in pain responses have been shown with conflicting results [3], this effect was not dependent on the age of the subjects, as it was observed both in young and old animals. Taking into consideration the short period mediating the three tests $(2 \mathrm{~min})$, it becomes evident that the mechanisms responsible for the phenomena cannot be directly ascribed to corticosteroid receptor activation (which involves activation of nuclear receptors). Alternatively, the sustained hypercorticalism is likely to alter other neurotransmitter systems/levels. Amongst these opioids are a likely candidate, as was shown in a potentiation of morphine analgesia (TF and hot-plate tests) in rats given inescapable shock prior to pain measurements but not in animals exposed to escapable shocks or restraint stress [17]. These findings confirm that uncontrollable stress enhances morphine analgesia [7]. Thus, if inescapable shock leads to opioid release and subsequent analgesia, a similar mechanism might occur in our stressed animals: more specifically, the first exposure to the TF test may trigger an abnormally high release of opiates within the CNS in stressed animals, and the re-exposure to the test in the next 2 min would still occur under an analgesic state, thereby accounting for the increased tolerance to painful stimuli. However, experiments involving the administration of opioid antagonists before the exposure to successive noxious stimulation are needed in order to test this hypothesis.

Evidence has recently shown that pain perception is the result of a fine balance between descending antinociceptive (inhibiting) and pronociceptive (facilitating) actions upon spinal nociceptive transmission. In fact, manipulations of different supraspinal pain control centers have revealed the existence of nuclei that inhibit or increase [9,12] pain behavior. Disruption of the neurotransmitter balance regulating the large network of connections between the different components of the supraspinal pain control system may lead to changes in pain perception in the model of chronic unpredictable stress used in this study.

Furthermore, it was proposed that morphological rearrangements in the brain structures (e.g. hippocampus) brought on by various types of allostatic load might influence the cognitive appraisal of the pain response [11]. While previous studies showed a stress-induced structural reorganization of the hippocampal formation, the present study confirms that pain threshold is altered in chronically stressed subjects. These findings suggest that as a consequence of indirect long-term activation of the HPA axis by stressful stimuli, central mechanisms of pain perception are likely to be impaired. Ultimately, a more detailed evaluation of the morphofunctional consequences of chronic stress and pain in the limbic system might improve the knowledge of the motivational-affective component of pain and help in the design of better therapeutic strategies for the many subjects currently suffering from chronic pain.

\section{Acknowledgements}

This study was supported by FCT project no. POCTI/NSE/46399/2002 (co-financed by FEDER) and the Grünenthal Foundation.

\section{References}

[1] W.C. Clark, J.C. Yang, M.N. Janal, Altered pain and visual sensitivity in humans: the effects of acute and chronic stress, Ann. N. Y. Acad. Sci. 467 (1986) 116-129.

[2] T. Crisp, J.L. Stafinsky, D.L. Hoskins, B. Dayal, K.M. Chinrock, M. Uram, Effects of aging on spinal opioid-induced antinociception, Neurobiol. Aging 15 (1994) 169-174.

[3] L. Gagliese, R. Melzack, Age differences in nociception and pain behaviours in the rat, Neurosci. Behav. Rev. 24 (2000) 843-854.

[4] G.D. Gamaro, M.H. Xavier, J.D. Denardin, J.A. Pilger, D.R. Ely, M.B. Ferreira, C. Dalmaz, The effects of acute and repeated restraint stress on the nociceptive response in rats, Physiol. Behav. 63 (1998) 693-697.

[5] C. Goicoechea, M.J. Ormazábal, M.J. Alfaro, M.I. Martin, Agerelated changes in nociception, behavior and monoamine levels in rats, Gen. Pharmacol. 28 (1997) 331-336.

[6] J.P. Herman, W.E. Cullinan, Neurocircuitry of stress: central control of the hypothalamo-pituitary-adrenocortical axis, Trends Neurosci. 20 (1997) 78-84.

[7] R.L. Hyson, L.J. Ashcraft, R.C. Drugan, J.W. Grau, S.F. Maier, Extent and control of shock affects naltrexone sensitivity of stress-induced analgesia and reactivity to morphine, Pharmacol. Biochem. Behav. 17 (1982) 1019-1025.

[8] A.E. Kalyuzhny, U. Arvidsson, W. Wu, M.W. Wessendorf, $\mu$-Opioid and $\delta$-opioid receptors are expressed in brainstem antinociceptive 
circuits: studies using immunocytochemistry and retrograde tracttracing, J. Neurosci. 20 (1996) 6490-6503.

[9] D. Lima, A. Almeida, The medullary dorsal reticular nucleus as a pronociceptive centre of the pain control system, Prog. Neurobiol. 66 (2002) 81-108.

[10] S. Mangiavacchi, F. Masi, S. Scheggi, B. Leggio, M.G. De Montis, C. Gambarana, Long-term behavioral and neurochemical effects of chronic stress exposure in rats, J. Neurochem. 79 (2001) 1113-1121.

[11] B.S. McEwen, Plasticity of the hippocampus: adaptation to chronic stress and allostatic load, Ann. N. Y. Acad. Sci. 933 (2001) 265-277.

[12] M.J. Millan, Descending control of pain, Prog. Neurobiol. 66 (2002) $355-474$.

[13] E.S. Onaivi, S. Payne, J.W. Brock, A. Hamdi, S. Faroouqui, C. Prasad, Chronic nicotine reverses age-associated increases in tail-flick latency and anxiety in rats, Life Sci. 54 (1993) 193-202.

[14] Z. Pavlovic, R.J. Bodnar, Antinociceptive and hypothermic crosstolerance between continuous and intermittent cold-water swims in rats, Physiol. Behav. 54 (1993) 1081-1084.
[15] W.A. Pedersen, R. Wan, M.P. Mattson, Impact of aging on stressresponsive neuroendocrine systems, Mech. Ageing Dev. 122 (2001) 963-983.

[16] N. Sousa, O.F.X. Almeida, F. Holsboer, M.M. Paula-Barbosa, M.D. Madeira, Maintenance of hippocampal cell numbers in young and aged rats submitted to chronic unpredictable stress. Comparison with the effects of corticosterone treatment, Stress 2 (1998) 237-249.

[17] L.C. Sutton, S.E. Lea, M.J. Will, B.A. Schwartz, C.E. Hartley, J.C. Poole, L.R. Watkins, S.F. Maier, Inescapable shock-induced potentiation of morphine analgesia, Behav. Neurosci. 111 (1997) $1105-1113$.

[18] G.W. Terman, M.J. Morgan, J.C. Liebeskind, Opioid and non-opioid stress analgesia from cold water swim: importance of stress severity, Brain Res. 372 (1986) 167-171.

[19] M. Zimmermann, Ethical guidelines for investigations of experimental pain in conscious animals, Pain 16 (1983) 109-110. 